\title{
Anabases
}

ANABASES Traditions et réceptions de l'Antiquité

18 | 2013

Varia

\section{Pythios/Pythes e la moglie da Erodoto a Plutarco}

Gabriella Vanotti

\section{(2) OpenEdition}

\section{Journals}

Edizione digitale

URL: http://journals.openedition.org/anabases/4310

DOI: 10.4000/anabases.4310

ISSN: 2256-9421

\section{Editore}

E.R.A.S.M.E.

\section{Edizione cartacea}

Data di pubblicazione: 1 ottobre 2013

Paginazione: 11-32

ISSN: 1774-4296

Notizia bibliografica digitale

Gabriella Vanotti, «Pythios/Pythes e la moglie da Erodoto a Plutarco », Anabases [Online], 18 | 2013,

Messo online il 01 novembre 2016, consultato il 21 octobre 2019. URL : http://

journals.openedition.org/anabases/4310 ; DOI : 10.4000/anabases.4310 


\section{Pythios/Pythes e la moglie da Erodoto a Plutarco}

Gabriella VANotTi

\section{Pythios in Erodoto}

ERODOTO Nel CORSO del VII libro, descrivendo Serse in marcia con il proprio esercito verso Sardi, riferisce un evento dai risvolti paradigmatici e tragici nello stesso tempo, di cui si rese protagonista il lido Pythios, figlio di Atys. La vicenda, che occupa i capitoli 27-29 e 38-39, ha tutti i connotati per qualificarsi come short story o novella, secondo le definizioni in uso da parte della critica anglosassone per qualificare i brevi racconti, che arricchiscono, nella loro peculiarità contenutistica e intertestuale, la complessiva trama narrativa delle Storie ${ }^{1}$.

La comparsa in scena del Lido coincide con l'arrivo di Serse in Frigia; infatti, egli accorse nei pressi di Celene, non lontano dalle sorgenti del fiume Meandro, ad accogliere ospitalmente il gran re e il suo esercito in marcia verso l'Ellesponto e la Grecia, offrendo di provvedere, con grande munificenza, al vettovagliamento dei militari e al finanziamento dell'intera impresa.

Agli occhi di gran parte della critica $^{2}$ il dovizioso Pythios, figlio di Atys, risulta identificabile con il nipote di Creso, che, in effetti, come è ben noto, fu padre dello sfor-

1 Sul posto delle short stories nell'economia delle Storie erodotee vd. di recente V. GraY, «Short stories in Herodotus' Histories», in E.J. Baker, I.J.F. De Jong, H. van WeEs (edd.), Brill's Companion to Herodotus, Leiden-Boston-Köln, 2002, p. 291-317. Importanti osservazioni, inoltre, in I.J.F. DE JoNG, "Narrative Unity and Unity», ibidem, p. 245-266.

2 Di questa opinione già W.W. How, J. Wells, A Commentary on Herodotus, II, Oxford, 1912, p. 138; R.W. MacAN (ed.), Herodotus. The Seventh, Eighth and Ninth Books, 
tunato Atys, morto a causa di Adrasto e della fatale caccia al cinghiale ${ }^{3}$. L'appartenenza alla stirpe dei Mermnadi, in effetti, ben si attaglia, oltre che alla provenienza geografica, soprattutto all'immensa ricchezza vantata da Pythios, che, fra l'altro, come informa Erodoto ${ }^{4}$, qualche tempo prima dei fatti esposti avrebbe offerto in dono al gran re Dario il famoso platano e la vite d'oro, opere d'arte mirabili, eseguite, secondo una tradizione ben attestata, dall'artista samio Teodoro ${ }^{5}$.

Nella pagina erodotea l'iniziale incontro fra Serse e Pythios sembra svolgersi in un clima di cordiale fair play, visto che i due uomini non mancarono di scambiarsi gesti di stima e di munificenza, quasi sfidandosi in una sorta di gara di reciproca generosità, arrivando l'uno, il Lido, a mettere a disposizione tutti i suoi averi per l'impresa bellica persiana; rifiutando l'altro, il gran re, di accogliere l'offerta e, anzi, impegnandosi personalmente ad accrescere le ricchezze dell'ospite.

Dunque il comportamento di Pythios sembrerebbe, in apparenza, improntato alla fedeltà e alla sottomissione nei confronti del proprio sovrano, mentre quello di Serse parrebbe dettato dalla riconoscenza e, insieme, dal compiacimento nei confronti del suddito fedele, al cui generoso donativo, peraltro, non è concessa accoglienza, quasi a sottolineare l'indiscussa e indiscutibile primazia regale. In realtà, il tono fin troppo irenico dell'esposizione erodotea dovrebbe indurre il lettore, memore delle tragiche vicende in cui si trovarono coinvolti su fronti opposti i rispettivi avi dei protagonisti, Ciro e Creso, a sospettare che la esagerata munificenza del Lido fosse determinata dal prudente proposito di stornare da se stesso e dalla propria famiglia qualunque atto di ira e di violenza regale, cui già i suoi antenati in passato erano stati sottoposti ${ }^{6}$.

Ma, come vedremo, allo spirito inizialmente previdente di Pythios fece seguito un comportamento, nella realtà fattuale, assai maldestro, destinato a culminare in un atroce epilogo, del quale forse è possibile ravvisare un'anticipazione nel fugace riferi-

London, 1908, p. 41. Per un inquadramento recente del personaggio vd. S. LEWIS, «Who is Pythius the Lydian?», Histos 2 (1998), p. 1-5, con citazione della precedente bibliografia. Ulteriori indicazioni sul personaggio infra.

3 Herodotus I, 34-43. Poiché Erodoto riferisce che Atys morì subito dopo essersi ammogliato, bisogna supporre, forse un po' forzatamente, che Pythios sia stato concepito all'indomani delle nozze e che non abbia potuto conoscere il proprio padre; così propone LEWIS, "Who is Pythius», p. 3. Sul significato della presumibile appartenenza di Pythios alla dinastia mermnade nella architettura espositiva erodotea vd. infra.

4 Herodotus VII, 27, 2. Cfr. Plinius, Naturalis historia, XXXIII, 137 (su cui vd. infra).

5 Athenaeus XII, 514F-515A. Le notizie sulle mirabili opere e sulle relative fonti letterarie sono raccolte da MACAN, Herodotus, p. 42; e da P. BRIANT, Histoire de l'empire perse: de Cyrus à Alexandre, Paris, 1996, p. 248. I manufatti, fabbricati per il re Creso (come conferma Plinius, Naturalis historia, XXXIII, 51), potrebbero essere giunti in eredità a Pythios da Creso, che li avrebbe ceduti in dono a Dario.

6 Pythios è definito "mirror image of Croesus» da R. ThOmas, «Herodotus and Eastern Myths and Logoi: Deioces the Mede and Pythius the Lydian», in E. Baragwanath, M. DE BAKKer (edd.), Myth, Thruth, and Narrative in Herodotus, Oxford, 2012, p. 243. 
mento erodoteo alle sventure del satiro Marsia, evocate in occasione dell'incontro fra Pythios e Serse a Celene. Secondo lo storico, infatti, proprio nella piazza della città frigia era esposta la pelle del satiro. Dunque nello stesso luogo, in cui si conservava memoria della pena inflitta da Apollo al suo arrogante e stolto sfidante nelle gare liriche, si compì la vanitosa offerta da parte di Pythios di una cospicua somma di denaro al gran re; ma, come il dio ebbe facilmente a superare il proprio avversario nella performance musicale, così Serse surclassò il donativo di Pythios con un'offerta in denaro ancora più generosa. E non è tutto. L'evocazione del terribile scorticamento, inflitto da Apollo al vivo Marsia, per punirne l'arroganza, in qualche modo, sembra anticipare l'orrenda mutilazione perpetrata, come si vedrà, sul corpo del figlio di Pythios dal potente sovrano persiano.

Sulle vicende del maggiorente lido Erodoto torna a soffermarsi, dopo aver descritto le imponenti opere ingegneristiche eseguite dall'esercito del gran re per consentire il traghettamento della fanteria persiana dall'Anatolia in Europa. A questo punto, come si osserverà, lo storico non evita di rimarcare con chiarezza la dabbenaggine dell'ingenuo Pythios.

Il Lido, sicuramente ben informato sugli esiti nefasti cui era andata incontro la primitiva costruzione di ponti sui Dardanelli e soprattutto sull'eclissi solare, che si era verificata in occasione dei lavori sull'Ellesponto e presso il monte Athos ${ }^{7}$, aveva con perspicacia intuito come tali eventi fossero forieri di cattivi presagi per l'intera spedizione persiana; del resto il suo stesso onomastico e la sua possibile appartenenza alla casata dei Mermnadi ne suggerisce il rapporto di familiarità con l'arte mantica, quella delfica in particolare ${ }^{8}$.

Pertanto Pythios, confidando irrealisticamente e ingenuamente nell'amicizia, che presumeva di aver ormai stabilmente contratto con il gran re, si presentò al suo cospetto, per perorare l'esenzione del maggiore dei suoi cinque figli dai doveri bellici, senza tener conto della smisurata tracotanza e dell'ira violenta, che il sovrano non avrebbe mancato di manifestare di fronte a una simile richiesta; essa implicitamente comportava un giudizio di sfiducia sulla spedizione militare in corso e pure, in definitiva, sulla persona e sull'operato regale. Di fatto, il Lido non solo non si avvide di non saper celare i suoi riposti timori, ma neppure diede il giusto peso alla sua condizione di sudditanza nei confronti del proprio sovrano, che, invece, già in precedenza, l'aveva voluta ben notificare, rifiutandosi, come si è detto, a Celene, di accettarne il donativo in denaro; e, anzi, arrivando a integrare l'ammontare del patrimonio del suddito con i

7 L'eclissi evocata da Erodoto sarebbe fantasiosa, secondo LEWIS, "Who is Pythius», p. 2 e n. 6. In proposito vd. anche How, Wells, A Commentary, p. 139.

8 Che la stirpe dei Mermnadi fosse particolarmente legata all'oracolo delfico si evince soprattutto dal racconto erodoteo della storia di Creso, come è ribadito da LEWIS, «Who is Pythius», p. 1-3. 
propri averi ${ }^{9}$. E Pythios neppure tenne conto dell'incontenibile violenza di Serse, che, qualche tempo prima, era arrivato, a detta di Erodoto ${ }^{10}$, a percuotere l'Ellesponto con trecento colpi di verga e a rivolgere alle acque del mare parole oltraggiose, per aver in qualche modo ostacolato il prosieguo della sua impresa bellica.

Peraltro il gran re fu indotto a giudicare oltraggiosa la richiesta del Lido anche dalle rassicuranti, ma mendaci e fuorvianti parole dei Magi: costoro, per pura piaggeria, avevano riferito come l'eclissi solare lasciasse presagire l'abbandono delle loro città da parte dei Greci, anziché l'irreparabile sconfitta persiana. Pertanto Serse ${ }^{11}$ non esitò a rimproverare aspramente Pythios per non aver confidato nel valore bellico del proprio re, mettendosi al suo seguito con l'intera famiglia, moglie compresa ( $\tau$ òv $\chi \rho \tilde{\eta} v$

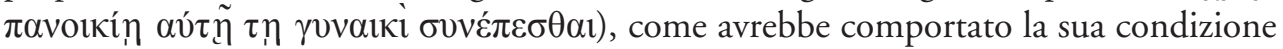
di suddito, e di aver, invece, perorato la causa del figlio maggiore. Per tanto non mancò di infliggergli un terribile fio, intimando che questo figlio fosse trucidato e che le due metà del suo corpo facessero ala al passaggio dell'esercito persiano ${ }^{12}$. Infine, l'implaca-

9 Che il rapporto fra Serse e Pythios sia impostato da Erodoto sulla base della reciprocità, alla quale il Lido sarebbe venuto meno, chiedendo la salvaguardia del proprio figlio maggiore, è sostenuto da ThOMAS, «Herodotus» p. 244. Sull'importanza del concetto di reciprocità nelle Storie di Erodoto vd. le decisive osservazioni di J. Gould, Herodotus, London, 1989 (Bristol Classical 2002), p. 42-85.

10 Herodotus VII, 35. L'insensato gesto di Serse sarrebbe stato frutto della sua incontenibile irascibilità (come lascerebbe intendere lo stesso Herodotus VII, 54, 1-3), oppure si trattava di un gesto rituale? Caute considerazioni sulla questione in H. SANCISI-WeERdenburG, "The Personality of Xerxes, King of Kings», in Baker, De Jong, van Wees (edd.), Brill's Companion, p. 579-590, part. p. 585, con referenze bibliografiche.

11 Sulla complessa personalità di Serse nel testo erodoteo, oltre al sopra citato lavoro di Sancisi-Weerdenburg, «The Personality of Xerxes», in Baker, De Jong, van Wees (edd.), Brill's Companion, p. 579-590, vd., in precedenza, K.H. WaTERS, Herodotus on Tyrants and Despots: a Study of Objectivity, Wiesbaden (Historia Einzel. 15), 1971, p. 65-85; e, di recente, M. Flower, "Herodotus and Persia», in C. Dewald, J. Marincola (edd.), The Cambridge Companion to Herodotus, Cambridge, 2009, p. 274-289, part. 282-283; E. Baragwanath, Motivation and Narrative in Herodotus, Oxford, 2008, p. 240-265, con ricca discussione della copiosa bibliografia precedente.

12 Che il tremendo destino di morte inflitto al figlio maggiore di Pythios rappresenti un feroce rituale bellico di matrice orientale è stato sostenuto da molti studiosi moderni, fra gli altri, da How, Wells, A Commentary, p. 145; Waters, Herodotus on Tyrants, p. 75 e n. 74; T. Harrison, «The Persian Invasions», in Baker, De Jong, van Wees (edd.), Brill's Companion, p. 551-578, part. p. 576 e n. 64. Sottolinea la valenza apotropaica assegnata da Serse al macabro squartamento del giovane J.A. Evans, «The Story of Pythius», in ID., The Beginnings of History: Herodotus and the Persian Wars, Campbelville, 2006, p. 85-87 (=Liverpool Classical Monthly, 13 [1988] p. 139), nonché BRIANT, Histoire de l'empire, p. 255, con ulteriori riferimenti bibliografici. Ritiene che Erodoto, a conoscenza di un rituale di origine medio orientale (forse ittita) lo abbia rielaborato, adattandolo al proprio contesto narrativo R. Rollinger, «Herodotus and the Intellectual Heritage of the Ancient Near East», in S. Aro, R.M. Whiting (edd.), The Heirs of Assyria, Helsinki, 2000, 
bile sovrano non si astenne dall'umiliare sino in fondo il misero Pythios ${ }^{13}$, affermando sarcasticamente di avergli comminato un castigo inferiore a quello che avrebbe meritato per la sua improvvida tracotanza, dal momento che concedeva a lui e ai quattro figli minori di continuare a vivere ${ }^{14}$. Dunque, per la seconda volta, il gran re si produsse in un gesto in apparenza evergetico, ma, in realtà, dai risvolti macabri e dai toni sadici, nei confronti del proprio sottomesso.

Erodoto null'altro riferisce a proposito di Pythios e del suo destino, dopo aver descritto la morte dell'infelice primogenito. In complesso risulta plausibile credere che la vicenda del Lido, pur configurandosi come non del tutto irreale ${ }^{15}$, sia stata ri-pensata e ri-costruita dallo storico per essere inserita, in quanto short story, entro una più ampia trama narrativa, di cui anticipa e conferma i risvolti moraleggianti. Attraverso il macabro racconto, infatti, Erodoto non manca di sottolineare i nefasti auspici sotto i quali prendeva avvio la campagna di Serse; non a caso, a distanza di qualche capitolo, quasi a siglare gli eventi precedentemente esposti, viene attribuita al saggio Artabano ${ }^{16}$,

p. 65-83, con ricca discussione della precedente bibliografia. Su tale punto di vista vd. ora le considerazioni di ThOmAS, «Herodotus», p. 233-253. La studiosa (p. 238) ritiene possibile che altri Greci, prima di Erodoto, avessero già offerto una rilettura in chiave ellenica della "storia" persiana: «It therefore seems probable that there are further stages of tradition and storytelling to be considered in the creation of this tale of Pythius».

13 Ritiene che la severa risposta di Serse non sia dettata dal crudele desiderio di umiliare Pythios, ma dal giusto intento di punire l'uomo, che, divenuto suo philos, si era dimostrato incapace di gestire questo rapporto privilegiato con il proprio sovrano BARAGWANATH, Motivation, p. 274-276. Sul tema della punizione nelle Storie di Erodoto W. Desmond, «Punishments and the Conclusion of Herodotus' Histories», Greek Roman and Byzantine Studies, 44 (2004), p. 19-40.

14 Come è stato più volte segnalato dalla critica, una vicenda non dissimile è raccontata in Herodotus IV, 84. In questo caso si parla del persiano Oiobazo, che chiese a Dario di esentare uno dei suoi tre figli dalla spedizione scitica. Il gran re, per tutta risposta, li fece uccidere tutti.

15 Sono dell'avviso che la figura e la vicenda di Pythios, pur presentando risvolti di sapore moraleggiante, siano in buona parte reali LEWIS, "Who is Pythius», p. 2; e, già in precedenza, Macan (ed.), Herodotus, p. 41; di questo parere, di recente, anche Rollinger, «Herodotus», p. 66, con ricca rassegna bibliografica. Più scettico sul fatto che la vicenda presenti particolari storici How, Wells, A Commentary, p. 145. Sulla ri-elaborazione erodotea di questa story vd. ora THOMAS, «Herodotus», p. 233-253; sulla ri-elaborazione da parte di Erodoto di miti, storie, tradizioni, in generale, vd. A. GRIFFITHS, «Stories and Storytelling in the Histories», in Dewald, Marincola (edd.), The Cambridge Companion, p. 130-144. Sulle fonti orali e scritte di Erodoto S. Hornblower, "Herodotus and His Sources of Information", in Baker, De Jong, van Wees (edd.), Brill's Companion, p. 373-386, con discussione della precedente bibliografia.

16 Artabano aveva già tentato di contenere la hybris di Serse, ammonendolo con parole sagge, secondo Herodotus VII 10, 2. Sul ruolo di saggio consigliere, assunto dall'influente persiano nelle Storie, vd., fra gli altri, WATERs, Herodotus on Tyrants, p. 70-75; e soprattutto R. Lattimore, "The Wise Adviser in Herodotus», Classical Philology, 34 
zio del sovrano, la seguente affermazione dal valore gnomico, ma anche vagamente profetico: "Così la morte, essendo la vita piena di affanni, è per l'uomo il rifugio di gran lunga preferibile; e il dio dopo avergli fatto assaggiare la dolcezza della vita, si rivela invidioso verso di lui ${ }^{17}$ ". Le parole di Artabano richiamano un tema (di sapore soloniano) caro a Erodoto: quello dell'incertezza della sorte umana: esse dovrebbero suonare di monito per Serse, inducendolo a ridimensionare la sua esagerata superbia e la sua violenza. Al contrario, il re persiano, lungi dal seguire il saggio consiglio, non mancò, in seguito, di costellare la propria intera campagna di episodi violenti, chiudendola con un atto efferato, che, per certi versi, richiama la vicenda di Pythios, al punto che si è supposto che entrambe le short stories fossero state concepite per fungere da cornice all'intera impresa bellica ${ }^{18}$. Come nella storia di Pythios, infatti, anche in questo episodio conclusivo Erodoto ${ }^{19}$ registra nel comportamento di Serse il succedersi di una sanguinosa e irragionevole vendetta a un'iniziale e ingannevole benevolenza, visto che, lasciata la Grecia, sconfitto e umiliato, egli si sarebbe imbarcato su una nave fenicia, destinata a incorrere in una violenta tempesta. Allora, fattosi timoroso per la sua stessa vita, avrebbe costretto una parte dei passeggeri a gettarsi in mare, per evitare che l'imbarcazione affondasse. In fine, giunto sano e salvo a terra, dapprima volle donare al timoniere della nave una corona d'oro, in segno di apparente gratitudine per avergli consentito di far ritorno in Asia; ma, in seguito, lo fece uccidere implacabilmente, dopo averlo giudicato colpevole della morte degli sfortunati compagni di viaggio, soppressi, in realtà, per volontà regale.

Ma la storia di Pythios non è richiamata in discussione dallo storico di Alicarnasso all'unico scopo di focalizzare l'attenzione del lettore sulla insensata crudeltà di Serse.

(1939), p. 24-35; M. MogGi, "Artabano in Erodoto», in M. Giangiulio (ed.), Erodoto e il «modello erodoteo»: formazione e trasmissione delle tradizioni storiche in Grecia, Trento, 2005, p. 193-214. Sul tema della hybris in Erodoto vd. le osservazioni di D. Asheri in Erodoto. Le storie Libro I. La Lidia e la Persia (a cura di D. Asheri), Milano, 1988, p. XLVI-XLVIII.

17 Herodotus VII, 46, 4. Che le parole pronunciate da Artabano lascino evidenziare un debito erodoteo dal genere tragico è stato sostenuto, o negato dalla critica, in proposito, con non identici punti di vista, vd. WaTers, Herodotus on Tyrants, p. 86-100; e i più recenti lavori di S. SAÏD, «Herodotus and Tragedy», in BAKER, De Jong, van WeES (edd.), Brill's Companion, p. 117-147; e di A. Griffin, "Herodotus and Tragedy», in Dewald, Marincola (edd.), The Cambridge Companion, p. 46-59, con ricca discussione della precedente bibliografia; infine per un riesame complessivo della questione vd. ora R. Rutherford, "Tragedy and History», in J. Marincola (ed.), A Companion to Greek and Roman Historiography, 2, Oxford, 2007, p. 504-514.

18 Di questo parere, fra gli altri, GrAY, «Short stories in Herodotus' Histories», p. 295-296 e 304.

19 Herodotus VIII, 118, 1-4. Un accostamento fra questa vicenda e quella di Pythios era già stata proposta da D. LATEInER, The Historical Method of Herodotus, Toronto-BuffaloLondon, 1989, p. 154 ; S. Flory, The Archaic Smile of Herodotus, Detroit, 1987, p. 59-60. Va sottolineato che Erodoto giudica l'intera vicenda del tutto incredibile (VIII 119, 1). 
Come le altre short stories, essa evidenzia la complessa architettura del racconto erodoteo, all'interno del quale funge, come è stato osservato ${ }^{20}$, da mirror text, opportunamente inserito nella trama narrativa per meglio chiarificarne il senso complessivo. Pertanto non è da escludere, a nostro avviso, che l'infelice destino del maggiorente lido e dei suoi figli sia stato evocato anche allo scopo di ricordare come sulla loro misera fine continuassero a pesare la colpa del sanguinario avo Gige e la cattiva sorte toccata alla sua intera discendenza ${ }^{21}$. In tal senso le due stories, quella di Gige e quella di Pythios, entrambe concepite in chiave moraleggiante, potrebbero costituire il punto di partenza e il punto di arrivo dell'intermittente resoconto dedicato da Erodoto ad ascesa e caduta della dinastia mermnade; in altre parole, con l'atroce morte del figlio di Pythios si concluderebbe la terribile nemesi provocata dal sanguinario (suo malgrado) Gige ${ }^{22}$. Per questo, forse, non è del tutto casuale che nelle due stories ricorra un richiamo alle orecchie, giudicate fonti di informazione insidiose: nel caso di Gige, in quanto incapaci di documentare la bellezza della regina in modo così certo da non costringere l'infelice guardia regale a constatarla con i propri occhi; nel caso di Pythios, in quanto tralatrici di notizie sgradevoli. Infatti, come sostiene Serse, rivolgendosi al suddito lido: «l'animo degli uomini abita nelle orecchie ed esso, quando ascolta cose favorevoli, riempie di gioia il corpo, quando ascolta il contrario si gonfia d'ira ${ }^{23}$."

20 GraY, «Short stories in Herodotus' Histories», p. 305-6.

21 Di questa opinione, LEWIS "Who is Pythius», p. 1-5. Utili considerazioni sull'atteggiamento di Erodoto verso la dinastia mermnade e soprattutto verso il paradigmatico ruolo di Creso in M. Lombardo, «Erodoto storico dei Lidi», in O. Reverdin, B. Ganger (edd.), Hérodote et les peuples non grecs. Entretiens sur l'antiquité classique XXXV, VandoeuvresGenève 1990, p. 171-203; P. BRIANT, «Hérodote et la société perse», ibidem p. 69-104;

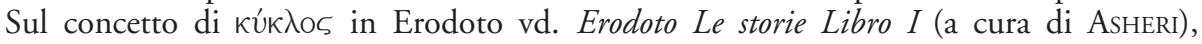
p. XLIII-XLVIII. Utili considerazioni sulle interazioni e intersezioni nel testo erodoteo in A. Beltrametti, Erodoto: una storia governata dal discorso. Il racconto morale come forma della memoria, Firenze, 1986, p. 111-130. La dabbenaggine di Pythios ricorda per certi versi quella di Creso: entrambi, infatti si qualificano come troppo confidenti nelle loro ricchezze e nella fortuna che queste ultime potrebbero loro garantire. Mettono in evidenza le numerose interconnessioni fra ascesa e caduta di Creso e dei Mermnadi e ascesa e caduta di Serse Gould, «Herodotus», p. 121-125; GRIFFIn, «Herodotus and Tragedy», p. 46-59; e più di recente BARAGWANATH, Motivation, p. 269-278. La studiosa cerca di evidenziare le numerose chiavi di lettura sottese all'episodio di Pythios, ben conscia del fatto che (p. 277) il metodo di Erodoto " is to leave readers to draw inferences themselves».

22 Herodotus I, 7-14. Il richiamo alla inattendibilità delle orecchie ricorre a I, 8, 2. Per una decodificazione di tale affermazione erodotea F. HARTOG, Le miroir d'Hérodote, Paris, 2001, p. 395-459. Sul tema opsis / akoévd. anche le considerazioni di C. Darbo-PesChansKi, Le discours du particulier. Essai sur l'enquête hérodotéenne, Paris, 1987, p. 84-88.

23 Herodotus. VII, 39, 1. Sul fatto che identiche parole o espressioni ricorrano all'inizio e al termine di una Ringkomposition insiste DE JonG, «Narrative Unity», p. 260. 


\section{Pythios dopo Erodoto: ovvero Pythes e sua moglie}

La vicenda, che vide protagonista Pythios, conobbe una certa fortuna nelle fonti successive a Erodoto. Ne troviamo traccia in due passi di età imperiale, l'uno risalente al De ira senecano ${ }^{24}$, l'altro alla Naturalis historia di Plinio ${ }^{25}$. Entrambi i testi non si discostano sostanzialmente dal racconto erodoteo, se non per particolari di scarso rilievo.

Seneca rimarca, come richiedeva il contesto in cui si trova inserito il racconto, gli aspetti moraleggianti della storia, sottolineando che i fatti violenti, in cui si produsse Serse, ne prepararono la sconfitta e la rovina.

Plinio, nella cui pagina il Pythios erodoteo viene denominato Pythes/Pythis e risulta originario della Bitinia ${ }^{26}$, appare soprattutto interessato a richiamarne in discussione la ricchezza. Infatti, non solo ricorda che donò il platano e la vite d'oro al re Dario, ma afferma pure che rifocillò e stipendiò per cinque mesi l'esercito di Serse, composto, a suo dire, niente meno che di 788000 uomini. Questi ultimi dati, estremamente puntuali, non compaiono nella versione erodotea e, trattandosi, per quanto ne sappiamo, di un unicum, potrebbero essere invenzione della penna pliniana, interessata a fornire cifre esorbitanti, allo scopo di enfatizzare la ricchezza del personaggio.

A distanza di qualche tempo, del dovizioso Pythes tornò a occuparsi Polieno ${ }^{27}$, in un contesto doppiamente peculiare, in quanto interessato agli stratagemmi e, in particolare, a quelli di matrice femminile. Come è noto, infatti, la parte finale dell'ottavo capitolo degli Strategemata è dedicato al racconto di quarantacinque astuzie messe in atto, individualmente o in gruppo, da donne audaci, per risolvere situazioni critiche di varia natura. E dunque è ovvio che l'autore si soffermi non solo sull'operato del maggiorente lido, ma anche e soprattutto su quello di sua moglie, della quale, come si è visto, praticamente, non si fa menzione, o quasi, nell'esposizione erodotea ${ }^{28}$.

La storia, nei termini in cui è riferita da Polieno, pare connotarsi come antecedente gli eventi bellici riportati da Erodoto, dei quali non si parla affatto. Il focus narrativo del testo stratagemmatico si incentra, invece, sulle miniere auree scoperte e acquisite da Pythes, in cui tutti i concittadini furono ben presto costretti a lavorare, abbandonando qualsiasi altra attività produttiva. Dall'esposizione emerge come l'avido

24 Seneca, De ira, III, 16, 4.

25 Plinius, Naturalis historia, XXXIII, 13.

26 Sulla confusione nei testi antichi fra una Pythopolis di Lidia e un'omonima città della Bitinia vd. infra, n. 29.

27 Polyaenus VIII, 42.

28 Nel testo erodoteo (VII, 39, 1) la moglie, come si è detto supra, è solo cursoriamente citata da Serse, per ricordare al proprio suddito che anch'ella avrebbe dovuto seguire la spedizione, in segno di fiducia nelle capacità belliche del sovrano. Sulle differenti tradizioni e sulla trasmissione della novella di Pythios/Pythes in Erodoto e nelle fonti successive vd. infra. 
uomo rivestisse, a quanto pare, un ruolo di prestigio nella città, come verosimilmente ne fosse il capo.

Non è difficile immaginare che, a causa dei suoi dissennati ordini, la vita e l'economia civiche avessero ben presto dato segni di crisi, al punto da indurre all'azione le donne locali; esse, a loro volta, invocarono e determinarono l'intervento della moglie di Pythes, cui Polieno assegna l'improbabile onomastico di Pythopolides. Si tratta di un unicum, che probabilmente si deve al desiderio di Polieno di gareggiare con il suo stesso illustre predecessore, Plutarco (che, come vedremo, aveva già trattato la vicenda nei Moralia $^{29}$ ), facendo sfoggio di informazioni in apparenza dotte, ma verosimilmente frutto della sua inventiva ${ }^{30}$.

Dunque, secondo Polieno, la donna non si fece pregare e ricorse a un raffinato stratagemma per indurre l'improvvido marito, accecato dalla bramosia di ricchezza, a ravvedersi. Ricorrendo all'abilità di esperti orafi, ella fece forgiare in oro splendide vivande e le imbandì al consorte, che, inizialmente entusiasta per la perfezione e lo splendore dei manufatti, frutto delle sue miniere, fu in seguito assalito dai morsi della fame. Allora, compresa la dissennatezza del proprio comportamento, permise ai cittadini di tornare a dedicarsi ad attività meno lucrative, ma più utili. Il richiamo alla leggenda del re Mida e al suo tocco d'oro è evidente ed è stato più volte evidenziato dalla critica ${ }^{31}$.

Non v'è dubbio che il racconto di Erodoto e quello di Polieno siano così divergenti che potrebbero persino riferirsi a due diversi personaggi, pressoché omonimi, vissuti persino in epoche diverse; infatti dal testo di Polieno non emerge alcun riferimento a eventi storici riconducibili all'invasione persiana, o comunque utili a definire la cronologia dei fatti. Entrambi gli autori concordano soltanto sullo stato patrimoniale della famiglia di Pythios/ Pythes. E tuttavia, lo storico di Alicarnasso, pur non mancando di rimarcare la cieca fiducia dell'uomo nel denaro, non ne ricorda la stolta avidità nello sfruttare il lavoro dei concittadini nell'attività mineraria. Tale particolare nella pagina

29 Plutarchus, Moralia, 262D-263C.

30 Evidentemente l'onomastico è tratto dal nome della omonima città Pythópolis. L'esistenza di una città, in Caria, così chiamata, e del relativo fiume Pythopolites è attestata da Stephanus Byzantinus s.v. Pythópolis. L'etnografo, che identifica l'antico centro con la recenziore località di Nv̂ $\sigma \alpha$, sita a sud di Sardi, nei pressi del corso del Meandro, mostra di conoscere anche la grande ricchezza del suo cittadino più famoso, Pythes, generoso elargitore di darici in oro all'esercito di Serse. Un villaggio chiamato Pithopolis e sito presso la palude Ascania era noto per le sue caratteristiche idriche all'anonimo autore del De mirabilibus auscultationibus (cap. 54). La localizzazione di questa palude (e quindi del finitimo villaggio di Pithopolis) in Bitinia rende forse ragione dell'insolita indicazione etnografica attribuita dal citato Plinio a Pythes (definito Pythis Bithynii), sulla base dell'omonimia dei due differenti centri.

31 Per tutti vd. P.A. Stadter, Plutarch's Historical Methods. An Analysis of the Mulierum Virtutes, Cambridge Mass., 1965, p. 122-123. 
di Polieno risulta, invece, essenziale, in quanto decisivo nel provocare l'intervento dell'intera comunità muliebre e il relativo proficuo stratagemma.

Giunge, però, definitiva conferma che il personaggio di Erodoto e quello di Polieno siano, in realtà, identificabili in un'unica persona da un testo di Plutarco, che, scrivendo circa un cinquantennio prima di Polieno, senza dubbio, fornisce la versione più articolata e, per certi versi, più eterodossa della tragica vicenda di Pythes, ben illustrando anche il ruolo svolto dalla di lui moglie.

\section{Pythes e la moglie nel trattato Mulierum virtutes di Plutarco}

La storia del Lido (che Plutarco denomina Pythes, come già in precedenza Plinio e in seguito Polieno ${ }^{32}$ ) e della consorte è riportata dal biografo al capitolo 27, l'ultimo del trattato Mulierum virtutes. Essa risulta scandita in tre segmenti narrativi, nel primo dei quali, in buona sostanza, sono riferiti i fatti esposti negli Strategemata di Polieno; nel secondo, in breve, le vicende narrate da Erodoto; nel terzo, infine, nuovi dettagli, riguardanti il ricco Lido, ma anche la moglie, attraverso i quali l'autore intende provare le qualità morali e il coraggio della donna, secondo quanto sostenuto nelle battute iniziali del trattato, ove si afferma, in polemica con l'autorevole Tucidide ${ }^{33}$, che: «una sola e identica è la virtù

32 La rispondenza fra il testo di Polieno e quello di Plutarco (si ricordi che, oltre alla nostra, ben diciannove delle ventisette storie riportate nel trattato plutarcheo ritornano nel settimo e nell'ottavo libro degli Strategemata di Polieno) ha indotto alcuni studiosi a postulare la dipendenza di quest'ultimo da Plutarco, ma altri a credere che i due autori abbiano recepito separatamente il loro racconto da una medesima fonte precedente. Discussione del problema (con una chiara affermazione dell'uso plutarcheo di prima mano di fonti svariate, risalenti anche a $\mathrm{V}$ e IV secolo e di una sostanziale dipendenza di Polieno da Plutarco) in STADTer, Plutarch's Historical Methods, p. 13-29 e soprattutto p. 126-140, seguito da M.T. SChetTino, Introduzione a Polieno, Pisa, 1998, p. 277-280; e più di recente da J. Boulogne, Plutarque. Conduites méritoires des femmes. Euvres Morales, IV, Paris, 2002, p. 29-38; a quest'ultimo studioso si rimanda per una puntuale discussione della precedente bibliografia. A una dipendenza di Plutarco e di Polieno da una fonte comune pensano, fra gli altri, invece, K. Ziegler, Plutarco, Brescia, 1965 (trad.

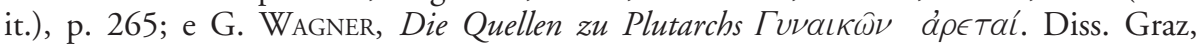
1967. Brevi riferimenti alla questione nel recente volume miscellaneo K. BRODERSEN (ed.), Polyainos. Neue Studien, Berlin, 2010, di cui risultano utili soprattutto i saggi di M. Pretzler, «Polyainos the Historian Stratagems and the Use of the Past in the Second Sophistic», p. 85-107; e di K. Brodersen, «Mannhafte Frauen bei Polyainos und beim Anonymus de mulieribus,, p. 149-159.

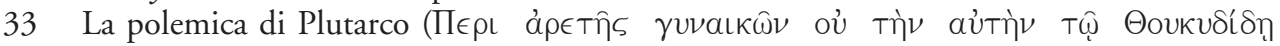
$\gamma \nu \omega \dot{\mu} \mu \nu)$ è diretta contro la famosa affermazione attribuita a Pericle da Tucidide (II, 45, 2). Lo storico ateniese, nell'epitafio pronunciato al termine del primo anno di guerra dallo statista, gli faceva dire: «Se è opportuno che io faccia riferimento anche alla virtù delle donne, di quelle donne, che d'ora in poi vivranno in stato di vedovanza, mi limiterò a un piccolo consiglio: grande è la vostra gloria se non tralignate dalla vostra natura e se fra gli 


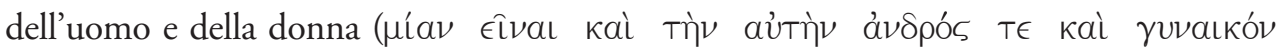

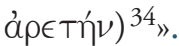

Per meglio illustrare tale assunto, nel caso di Pythes e della moglie, Plutarco intesse un serrato confronto fra $\mathrm{i}$ due coniugi, che risultano co-protagonisti della vicenda narrata, dato che il comportamento dell'uno/a serve a illuminare i risvolti psicologici della personalità dell'altro/a: in particolare, la dabbenaggine di Pythes e la sua scarsa lungimiranza (da cui, in parte, riesce tuttavia a riscattarsi nel finale della storia, come si vedrà) contribuiscono, attraverso un sottile gioco di synkrisis ${ }^{35}$, a rimarcare i meriti

uomini si parla di voi - della vostra virtù o delle critiche che vi si possono rivolgere - il meno possibile». Per un commento al passo tucidideo ancor utile P. Roussel, «Thucydide et la question féminine à Athènes», Revue des études grecques, 36 (1943), p. XII; inoltre vd., più di recente, i contributi di $\varnothing$. ANDERSEN, «The Widows, The City and Thucydides (II, 45, 2)», Symbolae Osloenses 42 (1987), p. 33-49; P. Cartledge, "The Silent Women of Thucydides: 2. 45. 2 Re-viewed", in R.M. Rosen J. Farrel (edd.), Nomodeiktes. Greek Studies in Honor of M. Ostwald, Ann Arbor 1992, p. 125-132; L. KalleT-MarX, "Thucydides 2. 45. 2 and the Status of War Widows in Periclean Athens», ibidem, p. 133-143; U. Fantasia, Tucidide. La Guerra del Peloponneso. Libro II, Pisa, 2003, p. 419-420. Per un confronto fra punto di vista tucidideo e plutarcheo vd. M. GarCía VAldés, "Plutarco versus Tucídides: Virtutes mulierum», in M. Jufresa, E. Mestre, P. Gomez \& P. Gilabert (edd.), Plutarc a la seva època: Paideia i societat, Barcelona, 2005, p. 297-312, con discussione della precedente bibliografia.

34 Plutarchus, Moralia, 242F. Che l'opera sia autentica è pressoché unanimemente sostenuto dalla critica. Vd. in proposito STADTER, Plutarch's Historical Methods, p. 1-2; e più di recente Boulogne, Plutarque. Conduites, p. 3, cui si rimanda per la discussione bibliografica. Sull'autenticità, fra l'altro, parebbe deporre il fatto che, per struttura e per contenuti, il trattato risulti sostanzialmente congruente con la produzione biografica plutarchea, della quale propone la tramatura espositiva. Per un inquadramento dell'opera all'interno del suo contesto culturale di riferimento, oltre a Boulogne, vd. K. O'BRIEN WiCKER, "Mulierum virtutes (Moralia 242E-263C)», in H.D. BETZ (ed.), Plutarch's Ethical Writings and Early Christian Literature, Leiden, 1978, p. 106-134 e di recente J. McInerney, «Plutarch's Manly Women», in R.M. Rosen, I. Sluiter, Andreia Studies in Manliness and Courage in Classical Antiquity (Mnemosyne suppl. 238), Leiden-Boston, 2003, p. 319-345.

35 Plutarco (Moralia, 243C) sostiene che «non c'è modo migliore di mettere in luce somiglianze e differenze fra virtù maschile e femminile che di mettere a confronto vite con vite, azioni con azioni...». Su tale affermazioni vd. le considerazioni di Boulogne, Plutarque. Conduites, p. 17-19. Sull'uso della synkrisis in Plutarco vd., fra gli altri, S. Swain, «Plutarchan Synkrisis», Eranos 90 (1992), p. 101-111; T.E. Duff, Plutarch's Lives Exploring Virtue and Vice, Oxford, 1999, p. 243-286; C. PELLING, "Synkrisis in Plutarch's Lives», in ID., Plutarch and History: Eighteen Studies, Swansea, 2002, p. 349-363; ID., "Synkrisis Revisited ", in A. PÉrez Jiménez, F. Titchener (edd.), Historical and Biographical Values of Plutarch's Works Studies Devoted to Professor P.A.Stadter, MálagaLogan, 2005, p. 325-340. 
della consorte, che, enfaticamente, già in apertura di discorso, è connotata come $\sigma o \phi n ́$ kaì $\chi \rho \eta \sigma т \eta^{36}$.

Ma l'interazione fra i due personaggi è, forse, a ben vedere, più complessa e raffinata di quanto potrebbe sembrare a una prima lettura. Pertanto vale la pena soffermarsi sul testo plutarcheo dei Moralia.

Come si è già detto, il racconto del biografo si apre con una prima sezione espositiva in cui ricorrono i medesimi dettagli riferiti da Polieno. Anche Plutarco, come l'autore degli Strategemata, ricorda, infatti, la straordinaria ricchezza del maggiorente lido, proprietario di molte miniere d'oro, nelle quali l'intera popolazione era stata costretta a forza a lavorare, lasciando negletta ogni altra occupazione. Di seguito il biografo descrive la reazione a tale dissennata imposizione da parte delle donne locali, che invocarono e determinarono l'intervento della moglie di Pythes. Questa prima iniziativa muliebre, collettiva, viene solo fugacemente tratteggiata e, senza dubbio, costituisce un'iniziale elogiativa presa d'atto, da parte del narratore, del coraggio e del buon senso dell'intera comunità femminile; in prosieguo, però, il focus narrativo risulta incentrato sull'operato individuale della sola moglie di Pythes.

Secondo Plutarco, dunque, costei imbandì per l'avido marito un sontuoso banchetto con vivande completamente forgiate in oro, augurandosi che i morsi della fame lo avrebbero indotto ben presto a dare una giusta misura alle cose.

Come risulta evidente, in questo primo segmento espositivo, il racconto non diverge da quello presente nel testo di Polieno e, a ben vedere, come Polieno, anche il biografo avrebbe potuto concludere a questo punto il proprio resoconto sulla $\dot{\alpha} \rho \in T \hat{n}$ della moglie di Pythes e delle sue provvide concittadine, risultando essa già ben illustrata fin da queste battute iniziali. Invece, nell'esposizione di Plutarco, questa sezione narrativa costituisce soltanto una sorta di lungo preambolo, che introduce al fulcro vero e proprio della vicenda, destinata a raggiungere l'apice, come vedremo, nella parte conclusiva della storia.

Quindi, la narrazione del biografo prosegue con un secondo segmento, di chiara derivazione erodotea: nell'esposizione dei Moralia risultano, infatti, assai brevemente condensati i capitoli del VII libro delle Storie ${ }^{37}$, discussi in apertura. Seguendo la versione dell'illustre predecessore, il biografo narra che Serse, ormai sul punto di invadere la Grecia, venne raggiunto da Pythes, intenzionato a elargire grandi doni a Serse, pur di ottenere l'esenzione di uno dei suoi numerosi figli dalla spedizione militare in corso, essendo ingenuamente convinto, ma a torto, di aver definitivamente guadagnato il favore regale.

Dall'esposizione plutarchea, come da quella erodotea, risulta fin troppo manifesto come la richiesta del Lido implicasse l'arrière pensée che l'impresa persiana fosse destinata a incorrere in un fallimento. E pertanto, a detta del biografo, Serse, avendosene

36 Plutarchus, Moralia, 262D.

37 Herodotus VII, 27-29; 38-40. 
a male, ordinò di uccidere all'istante uno dei figli di Pythes; mentre i restanti fratelli, qualche tempo dopo, morirono tutti in battaglia.

La menzione della scomparsa dei fratelli (non contemplata da Erodoto) assume un senso ben preciso nel testo plutarcheo, poiché costituisce l'ineludibile presupposto all'assunzione del potere politico da parte della loro madre, riferito in prosieguo di discorso. L'assenza del particolare nella pagina erodotea è altrettanto comprensibile: ben si concilia, infatti, con il disinteresse dello storico per le vicende di Pythios e della sua famiglia, successive alla morte violenta del figlio maggiore. Del resto, gli aspetti moralistici, ma anche le strategie narrative, sottese al racconto erodoteo, non sono i medesimi rintracciabili nell'esposizione plutarchea.

La storia di Pythios nel testo dello storico è quasi completamente affidata al discorso diretto, che in questo caso, come in altri analoghi, risulta particolarmente efficace per evidenziare "the imbalance of the power-relationship ${ }^{38}$ ». Niente di tutto ciò nel biografo, che costruisce il proprio racconto in chiave espositiva; inoltre, a differenza del predecessore, non mostra particolare attenzione agli eventi di guerra, né alla tirannia o alla crudeltà di Serse, né agli impliciti riferimenti all'inesorabile débacle cui egli era destinato, centrali, per contro, nella pagina erodotea ${ }^{39}$. E neppure recepisce la complessa e sofisticata trama intertestuale, che caratterizza le Storie, nelle quali la vicenda di Pythios fornisce spunto per ribadire al lettore, ancora una volta, amare valutazioni sull'incertezza del destino umano; si tratta di una sorta di meta-discorso ${ }^{40}$, che corre parallelo all'esposizione degli eventi, arricchendolo di molti spunti di riflessione. Nello specifico contesto del Mulierum virtutes, celebrativo della ả $\rho \in T \eta ́$ femminile, nessuno di tali temi morali o di tali soluzioni espositive doveva interessare particolarmente il biografo, che peraltro, come è ben noto, non poteva qualificarsi come pieno estimatore di Erodoto ${ }^{41}$.

38 La considerazione si deve a C. Pelling, «Speeches and Narrative in the Histories», in Dewald-Marincola (edd.), The Cambridge Companion, p. 103-111.

39 Sul punto di vista plutarcheo a proposito delle guerre persiane vd., fra i più recenti, J. Marincola, "Plutarch, "Parallelism" and the Persian-War Lives», in N. Humble (ed.), Plutarch's Lives: Parallelism and Purpose, Swansea, 2010, p. 121-143; E. Oudot, «Marathon, l'Eurymédon, Platées, laissons-les aux écoles des sopistes. Les guerres médiques au second siècle de notre ère», in P.L. Malosse, M.P. NoËL et B. Schouler (edd.), Clio sous le regard d'Hermès L'utilisation de l'histoire dans la rhétorique ancienne de l'époque hellénistique à l'Antiquité tardive, Alessandria, 2010, p. 143-157.

40 Così si esprime N. Luraghi, "Meta-historiē: Method and Genre in the Histories", in Dewald-Marincola (edd.), The Cambridge Companion, p. 77.

$41 \mathrm{Vd}$. in proposito soprattutto le osservazioni (per quanto concerne il De Herodoti malignitate) di J.P. Hershbell, "Plutarch and Herodotus - The Beetle in the Rose», Rheinisches Museum für Philologie, 136 (1993), p. 143-163; più in generale quelle di J. MARINCOLA, "Plutarch's Refutation of Herodotus», Ancient World, 25 (1994), p. 191-203; con prospettiva di lettura in parte diversa e più relativizzante, C. Pelling, "De malignitate Plutarchi Plutarch, Herodotus and the Persian Wars", in E. Bridges, E. Hall, P.J. Rhodes 
Veniamo, infine, alla terza e ultima sezione del racconto plutarcheo; essa è del tutto assente nella pagina erodotea, e pure in quella di Polieno ${ }^{42}$, mentre occupa un posto di grande rilievo nel testo del biografo; infatti, quanto esposto nei precedenti segmenti narrativi sembra finalizzato a inquadrare preliminarmente la personalità dei due co-protagonisti del racconto (Pythes e la moglie), per collocare nella giusta luce gli esiti finali della loro storia.

In questa terza sezione la personalità del Lido, già messa in discussione nelle precedenti battute del racconto per la stoltezza, di cui aveva dato prova nel governare i concittadini e nel rapportarsi al crudele Serse, viene ulteriormente censurata per l'incapacità mostrata (almeno in apparenza) nell'affrontare le avversità della vita. Al contrario, la figura femminile, pur restando per lo più in ombra sul piano narrativo, finisce per emergere sul piano morale per la evidente capacità di superare le avversità e di assumere, infine, su di sé le responsabilità familiari e poleiche, al punto da venire investita del governo della città.

Infatti, Plutarco ${ }^{43}$ riferisce che Pythes, colpito da grande dolore per la perdita dei

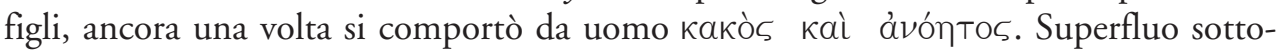
lineare il pressoché puntuale richiamo verbale alla breve e antitetica caratterizzazione morale della moglie, offerta in apertura di racconto: costei era stata definita, come si è

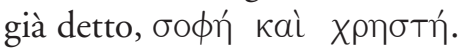

Ma torniamo alla condotta del marito: a detta del biografo, egli di fatto rinunciò a vivere, ma nello stesso tempo non ebbe il coraggio di togliersi drasticamente la vita. Per questo stabilì di rinchiudersi in una sorta di mausoleo, edificato al momento su un isolotto del fiume che bagnava la città, interrompendo ogni contatto con la realtà, ma chiedendo alla moglie di fornirgli di che nutrirsi per mezzo di una imbarcazione, finché non fosse per lui sopraggiunta la morte.

La menzione del mausoleo ( $\mu \nu \eta \mu \in \tilde{L} 0 \nu$ ) evoca il ricordo della tomba eroica, solitamente destinata ai leaders politici distintisi nella guida della loro città. Ma, nel caso di Pythes, il monumento si colora di tutt'altra valenza, poiché è, in realtà, il luogo nel quale l'uomo volle auto-segregarsi prima della morte, non certo per essere celebrato, ma, anzi, per estraniarsi dalla propria comunità e persino dalla propria famiglia, cui certamente non aveva recato benefici con il proprio operato. Dunque il Lido finisce per assumere, quasi paradossalmente, la connotazione di anti-eroe.

A questo punto la conclusione della vicenda, nella quale i due coniugi risultano ancora una volta affrontati in modo diretto: come riferisce Plutarco, l'uomo, dopo aver

(edd.), Cultural Responses to the Persian Wars Antiquity to Third Millenium, Oxford, 2007, p. 145-164. Utili osservazioni anche in BaragWanath, Motivation, p. 9-22.

42 L'assenza in Polieno di quest'ultima parte della vicenda di Pythes è giustificata dal fatto che in essa non sono contenuti riferimenti a stratagemmi orchestrati né da donne, né da uomini.

43 Plutarchus, Moralia, 263B. 
predisposto ogni cosa, trascorse quanto gli restava da vivere in una sorta di pesante abulia; la moglie, invece, per volere dello stesso marito, prese al suo posto il potere e

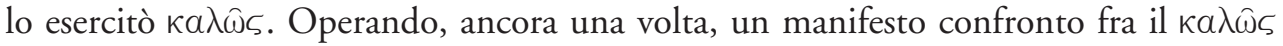

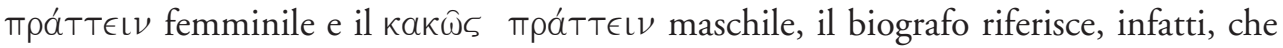
la donna riuscì a sovvertire tutte le inefficienze della precedente amministrazione, bene-

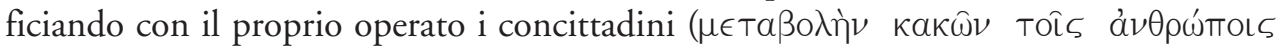
$\pi a \rho \hat{\epsilon} \sigma \chi \epsilon)^{44}$.

Non v'è dubbio che l'esposizione del biografo di Cheronea sia ben calibrata, sulla base di un equilibrato crescendo narrativo. Infatti, la definitiva rovina di Pythes, da un lato, e la funzione di spicco, anche di segno politico ${ }^{45}$, assunto dalla moglie, dall'altro, preannunciati, seppur fugacemente, sin dall'inizio della storia, si realizzano pienamente nella chiusa, comportando, almeno in apparenza, un sovvertimento dei rispettivi ruoli e una piena celebrazione dell' à $\rho \in T \eta ́$ femminile. Infatti, la donna, che nel primo segmento narrativo si era servita di uno stratagemma prettamente muliebre, per dissuadere il marito dal brutale sfruttamento dei sudditi nelle miniere aurifere, allestendogli un pranzo a base di vivande in oro ${ }^{46}$ e manifestando quindi in quell'occasione una

44 Ibid., 263C.

45 Tende a negare un ruolo politico alle donne plutarchee ritratte nel Mulierum virtutes P. Schmitt Pantel, «La religion et l'areté des femmes. À propos des vertus de femmes de Plutarque», in L Bodiou, V. MeHL (edd.), La religion des femmes en Grèce ancienne. Mythes, cultes et societé, Rennes, 2009, p. 145-159. La studiosa ritiene che molti episodi dell'opera siano incentrati sull'importanza del ruolo religioso delle donne. In questa sfera rientrerebbe anche il caso della moglie di Pythes, inizialmente indotta ad agire da una supplica di concittadine. Tuttavia, la studiosa riconosce che questa donna costituisce un'eccezione (p. 158), dal momento che, in conclusione della storia, sarà destinata a governare al posto del marito. Vd. inoltre P. Schmitt Pantel, «Femmes et héroïsme: un manque d'étoffe», in P. Schmitt Pantel (ed.), Aithra et Pandora. Femmes, Genre et Cité dans la Grèce antique, Paris, 2009, p. 179-192.

46 L'inversione di ruolo maschile/femminile, presente nella plutarchea vicenda della moglie di Pythes, richiama alla mente l'analoga e costante inversione di ruolo rivestita nel testo di Erodoto dalla principessa Artemisia nei confronti di Serse (VII, 99, 2-3;VIII, 68-69, 87, 2-88, 101-103). Con Artemisia la moglie di Pythes condivide, oltre che i natali microasiatici e il contesto cronologico, anche il destino di reggente del potere politico del marito deceduto e, in ultima analisi, la condizione vedovile, visto che Pythes rinuncia, ancor prima di morire, alla vita. Tuttavia, la figura femminile tratteggiata da Plutarco si colora di toni piuttosto diversi da quelli che caratterizzano l'erodoteo ritratto di Artemisia. L'impresa di

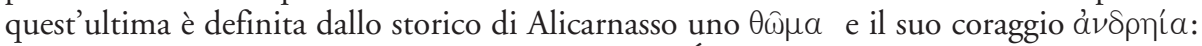
vd. R. WeIL, "Artémise ou le monde à l'envers», in Études offertes à A. Plassart, Paris, 1976, p. 215-224; A. Tourraix, "Artémise d'Halicarnasse, chez Hérodote, ou la figure de l'ambivalance», in M.M. Mactoux, E. GenY, Mélanges P. Lévêque, 5, Paris, 1990, p. 377-386; R. Vignolo Munson, «Artemisia in Herodotus», Classical Philology 7 (1988), p. 91-106; V. Sebillotte-Cuchet, «La fabrique d'une héroïne au Ve siècle: Hérodote et Artémise d'Halicarnasse», in Boudiou, MeHL (edd.), La religion, p. 19-32. Plutarco ricorre, invece, per la moglie di Pythes al più cauto vocabolo ả $\rho \in T \eta ́$, e, affermando che universalmente 
capacità di azione di sapore tutto femminile", nel III segmento sembra, a tutta prima,

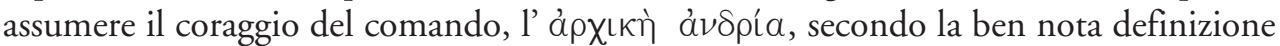
di Aristotele ${ }^{47}$, che giudica questa dote del tutto maschile. Per contro, il marito, sopraf-

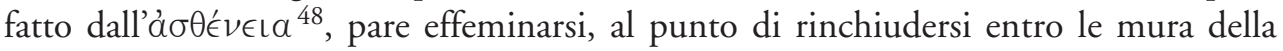
casa-mausoleo e di abbandonare i doveri della vita sociale e politica. E il sovvertimento delle rispettive funzioni sembrerebbe confermato pure dal fatto che l'uomo fosse il solo, per quanto afferma Plutarco ${ }^{49}$, ad affliggersi inconsolabilmente per la perdita dei figli, sentendosene responsabile, al punto di rinunciare a vivere. Quanto alla consorte, incredibilmente, non ne viene descritta la reazione alla terribile disgrazia.

Quest'ultimo particolare indubbiamente desta stupore, anche in considerazione della grande importanza che il biografo attribuisce alla cura materna dei figli. $\mathrm{Ne}$ dovremmo forse concludere che agli occhi di Plutarco ella finisca per assumere i connotati di mulier virilis ${ }^{50}$ ?

In realtà ci pare piuttosto che il disinteresse del narratore per la reazione femminile alla perdita dei figli risulti coerente con il ruolo complessivo che la donna riveste nell'economia del racconto: pur apparendo co-protagonista degli eventi narrati, tende a non assumere mai una sua piena e personale configurazione "realistica", ma si limita a essere un personaggio sostanzialmente artificioso, costruito per corrispondere alle esigenze argomentative plutarchee, come sembrerebbe confermare il suo assoluto anonimato, per certi versi, anch'esso stupefacente ${ }^{51}$.

A ben vedere, soltanto nel I segmento narrativo ella riveste i panni della protagonista, mentre nel III segmento, decisivo per la conclusione della vicenda, le vesti di primo attore toccano a Pythes. Costui, infatti, inizialmente definito incapace di morire,

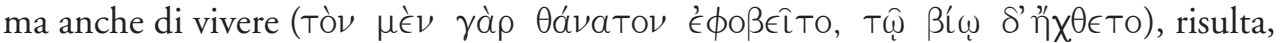
infine, in grado di dare disposizioni decisive per sé (di costruire il mausoleo nel quale auto-recludersi, di deviare il corso del fiume per creare una via di accesso al proprio

identica è la virtù di uomo e di donna, implicitamente lascia inferire che l'ả $\rho \in T \eta ́$ femminile non debba essere giudicata uno $\theta \omega \hat{\omega} \mu \alpha$; sul concetto di $\theta \omega \hat{\omega} \mu \alpha$ in Erodoto. R. Vignolo Munson, Telling Wonders: Ethnography and Political Discourse in the Work of Herodotus, Ann Arbor, 2001.

47 Aristoteles, Politica, 1260a 20.

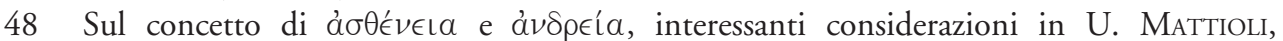

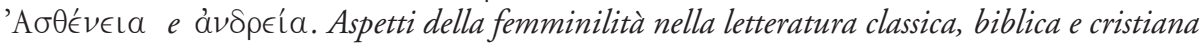
antica, Roma, 1983.

49 Plutarchus, Moralia, 263B.

50 Richiama l'attenzione sul fatto che la donna «virile», nella tradizione letteraria, risulti privata del ruolo materno, espressione, per eccellenza della sua femminilità, G. PETRONE, "La donna "virile"», in R. RAFFAELI (a cura di), Vicende e figure femminili in Grecia e a Roma, Ancona, 1995, p. 259-271.

51 L'anonimato femminile è motivo topico per la società greca del V secolo, come evidenziato, a suo tempo, da D. SCHAPS, "The Woman Least Mentioned: Etiquette and Women's Names», Classical Quaterly, 27 (1977), p. 323-330. 
eremo e per provvedere al proprio approvvigionamento alimentare), ma soprattutto per l'intera comunità, vale a dire di affidare il potere politico alla moglie, perché si prenda cura del benessere civico.

Si tratta di fatto della prima, per quanto tardiva, decisione saggia e proficua assunta dal maggiorente lido, il quale, pur rinunciando al proprio ruolo pubblico e persino alla propria vita, sa impartire alla moglie il fermo ordine finale di governare, imponendole di evitare ogni contatto con la sua persona, quasi a voler scongiurare le

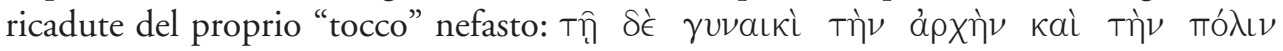

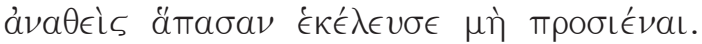

Quanto alla donna, se ne deve evincere che, nell'ottica di Plutarco, pur arrivando a reggere la città in luogo del marito, di fatto, non giunga a esercitare in modo pieno

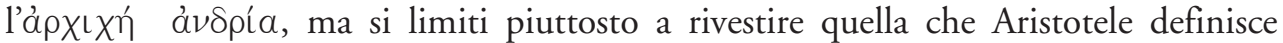

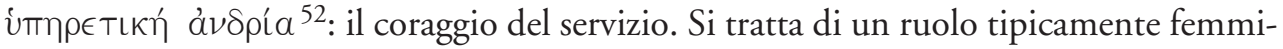
nile, che la moglie di Pythes, sicuramente non sprovvista di una sua personale synesis, assume per sostenere il coniuge, ma soprattutto per beneficare filantropicamente l'intera comunità civica. Dunque tutt'altro che una mulier virilis.

Come è stato proposto dalla critica $^{53}$, le donne descritte nel Mulierum virtutes possono essere suddivise fra passive e attive e queste ultime fra "dominanti" dominant women, donne, cioè, che agiscono per proprio tornaconto personale, spinte dall'ambizione o dalla sete di potere, e "supportanti", supportive women, donne, che sono guidate nelle loro azioni da nobili motivi, da sentimenti filantropici ${ }^{54}$. E come si è visto, la moglie di Pythes ha tutti gli attributi per appartenere alla categoria delle donne "supportanti", visto che è appunto la filantropia, piuttosto che l'ambizione personale o la sete di potere, a dettarne l'operato nei confronti dei concittadini, che in effetti benefica per ben due volte, sottraendoli, la prima, all'usurante ed esclusivo lavoro nelle miniere ed elevandone, la seconda, le condizioni di vita, dopo essere giunta alla guida della città.

Proprio a donne di tal fatta, a dire della critica, andrebbe il sincero elogio del biografo di Cheronea ${ }^{55}$.

52 Aristoteles, Politica, 1260a 20-23; 1277b 20-25.

53 Vd. in tal senso soprattutto K. BlomQvist, «From Olympias to Aretaphila: Women in Politics in Plutarch", in J. Mossman (ed.), Plutarch and His Intellectual World, London, 1997, p. 73-97. In realtà la classificazione delle donne virtuose di Plutarco è questione assai complessa, visto l'alto e variegato numero di figure femminili descritte nel trattato, impegnate non solo in azioni legate alla sfera del privato, ma anche in vere e proprie res gestae di indiscussa impronta politica. In ultima analisi è difficoltoso definire l'idea stessa di virtù femminile.

54 In buona sostanza si tratta di una classificazione non dissimile da quella proposta da

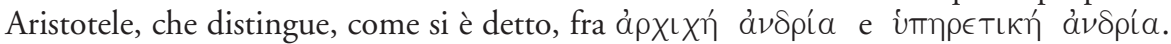

55 Vd. in tal senso BlomQVIST, «From Olympias to Aretaphila», p. 73-97. 


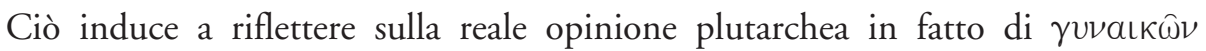
$\dot{\alpha} \rho \epsilon T^{56}$ e sull'affermazione dai toni "paritari”, con la quale si apre, in conclamata pole-

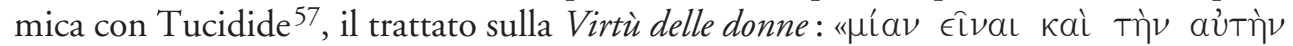

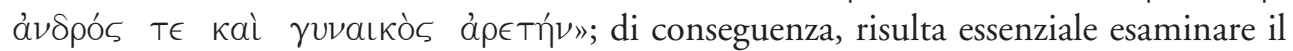
testo liberi dal preconcetto che l' autore, con esso, abbia atteso alla stesura di una sorta di manifesto femminista ${ }^{58}$.

Che il côté femminile suscitasse l'interesse plutarcheo è indubbio: lo dimostrano i titoli e i temi di alcuni fra i Moralia, quali i Coniugalia praecepta, l'Amatorius, l'An et mulier educanda est, di tutti forse il più significativo. Inoltre, non va sottovalutato che all'interno delle Vite non poche figure femminili sono campite a tutto tondo, con indubbia attenzione alla loro personalità, al loro operato e, in qualche caso, alla loro influenza politica ${ }^{59}$. E tuttavia, a ben vedere, dalla maggior parte delle opere menzionate, soprattutto dai Moralia, traspare un'attitudine tutt'al più paternalistica nell'affrontare i problemi legati all'universo muliebre: al sesso debole vanno senz'altro riservate la cura pedagogica e, nel matrimonio, il rispetto e la fedeltà del coniuge ma, all'interno della coppia, il primato maschile non viene mai messo in discussione ${ }^{60}$. In alcuni passi dei Coniugalia praecepta ${ }^{61}$ l'autore sottolinea addirittura la necessità

56 Sulla valenza del termine ả $\rho \in T n ́$ nel trattato plutarcheo vd. le giustre considerazioni formulate da O'Brien Wicker, "Mulierum virtutes, p. 112-113 e n. 24. La studiosa sostiene: «The concept of $\alpha \rho \in T \eta \eta n$ which Plutarch uses in the Mulierum virtutes embraces both the Homeric concept of courageous achievement and the notion of intellectual and moral excellence found in the philosophical tradition». In proposito vd. anche BouloGNE, Plutarque. Conduites, p. 8-9.

57 Thucydides II, 45, 2, su cui vd. supra n. 32.

58 Evidenzia i limiti del "femminismo" plutarcheo all'interno del trattato Boulogne, Plutarque. Conduites, p. 12-14. La medesima opinione era già stata espressa, in generale, da F. Le Corsu, Plutarque et les femmes, Paris, 1981, p. 270-274. Di lento riscatto della posizione etico-giuridica della donna greca parlano, commentando i Coniugalia praecepta, G. Martano, A. Tirelli (a cura di), Plutarco Precetti coniugali, Napoli, 1990, p. 13. Utile ed equilibrata sintesi della questione femminile in Plutarco in Y. VerniÈre, "Plutarque et les femmes», Ancient World, 25 (1994), p. 165-169; e di, recente, P. Schmitt Pantel, "Autour du traité de Plutarque Vertus de femmes (Gunaikôn Aretai)», CLIO. Histoire, femmes et societés, 30 (2009), p. 39-59. Con giudizio più critico P. WALCOT, «Plutarch on Women", Symbolae Osloenses, 74 (1999), p. 160-183.

59 Esame esaustivo di queste testimonianze in Le CORsu Plutarque, passim; e più di recente in G. D’IpPolito, «Il corpus plutarcheo come macrotesto di un progetto antropologico: modi e funzioni dell'autotestualità», in G. D'Ippolito, I. Gallo (edd.), Strutture formali dei Moralia di Plutarco, Napoli, 1991, p. 9-18, soprattutto p. 14-18; BlOMQVIST, «From Olympias to Aretaphila», p. 73-97.

60 Plutarchus, Moralia, 139C;140A-F;142E-F. L'autore afferma tra l'altro che l'uomo deve

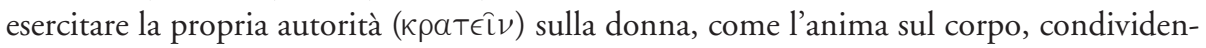
done i sentimenti e formando con lei una sola cosa (142E).

61 Plutarchus, Moralia, 142C-D. L'autore ricorda la scultura fidiaca dell'Afrodite degli Elei, rappresentata nell'atto di tenere sotto il piede una tartaruga: a suo dire, la statua 
che la donna non faccia sfoggio in pubblico non solo del proprio aspetto fisico, ma neppure del proprio eloquio; anzi, sostiene che sarebbe opportuno che si astenesse dal parlare, affidando, se necessario, al proprio coniuge il compito di manifestarne il pensiero. L'affermazione, in definitiva, non risulta molto difforme da quanto sostenuto da Tucidide a proposito delle donne ateniesi nell'epitaffio pericleo ${ }^{62}$. Ciò suggerisce che fra V secolo a.C. e II d.C. (fra Tucidide e Plutarco), in realtà, non molta acqua sia corsa sotto i ponti dell'emancipazione femminile ${ }^{63}$. In somma, a ragione, Plutarco può essere ritenuto più di altri generoso nel ridurre il profondo solco esistente fra uomo $\mathrm{e}$ donna nel mondo antico, e in quello greco in particolare, ma non può essere giudicato fervente sostenitore di una sorta di "corrente femminista", che intenda esaltare le qualità morali e intellettuali muliebri, contro l'opinione oscurantista diffusa nei secoli precedenti ${ }^{64}$.

Alla luce di queste considerazioni, assume consistenza l'ipotesi che molti dei temi

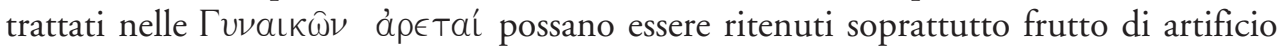
letterario ${ }^{65}$, tanto più che sono da considerarsi opera di circostanza, una sorta di consolatio, dedicata dall'autore all'amica Clea e da lei stessa sollecitata ${ }^{66}$, in occasione della

aveva un chiaro significato esortativo, quello di indurre le donne a restare in casa e a tacere (Plutarchus, Moralia, 142D). Per un'equilibrata analisi di questo trattato, oltre a Martano Tirelli (a cura di), Plutarco Precetti coniugali, passim; e a Plutarch's Advice to the Bride and Groom and A Consolation to His Wife (S.B. Pomeroy ed.), New YorkOxford, 1999, vd. C. Patterson, «Plutarch's 'Advice on Marriage': Traditional Wisdom through a Philosophical Lens», in $A N R W$ 2, 33, 6, 1992, p. 4709-4723; e più di recente, con analisi della bibliografia precedente, A.G. Nikolaidis, «Plutarch on Women and Marriage», Wiener Studien, 110 (1997), p. 27-88; J. Boulogne, «Plutarque et l'éducation des femmes», in M. Jufresa, E. Mestre, P. Gomez \& P. Gilabert (edd.), Plutarc a la seva època, p. 225-234.

62 Si tratta rispettivamente di Plutarchus, Moralia, 142C-D ; Thucydides II, 45, 2.

63 Così sostiene anche BlomQvist, «From Olympias to Aretaphila», p. 73-77. Va comunque ricordato che ulteriore, seppur cauto, apprezzamento della "virtù" femminile ricorre nell' Amatorius 767B-C, 769B.

64 Significativamente afferma: «La vision fugitive d'un monde où les femmes auraient fait preuve d'héroïsme n'est qu'une des facettes du discours grec sur l'inégalité entre les sexes.» Schmitt Pantel, "Autour du traité de Plutarque», p. 56.

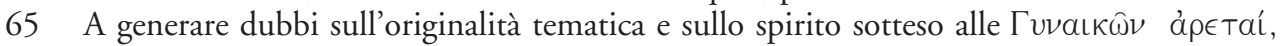
contribuisce il fatto che il trattato non qualifichi Plutarco come l'iniziatore di un nuovo genere letterario. Testi analoghi sulle virtù muliebri circolavano, soprattutto in ambito greco, almeno da età ellenistica. Dettagliata rassegna di tali trattati in STADTER Plutarch's Historical Methods, p. 3-5; D. GerA, Warrior Women. The Anonymous Tractatus de mulieribus, Leiden-New York-Köln, 1997, p. 29-30, 32-37; e ora in Boulogne, Plutarque. Conduites, p. 5-6.

66 Così sostiene lo stesso Plutarchus, Moralia, 242F. Parla di un'opera scritta rapidamente, priva di un vero filo conduttore, "au fil des souvenir et des notes de lecture» Boulogne, Plutarque. Conduites, p. 20. 
morte di Memmia Leontide, verosimilmente una parente, forse la suocera stessa della donna $^{67}$, e, come Clea, donna di grande cultura e preposta delle Tiadi di Delfi ${ }^{68}$.

Ma la questione, senz'altro complessa, richiederebbe altro e ben più ampio campo d'indagine, anche in considerazione della pluralità di personaggi muliebri, che, avvicendandosi nel trattato, offrono una vasta ed eterogenea gamma di sfumature in fatto di femminilità.

Qui interessa, in conclusione, piuttosto tentare di comprendere in che modo Plutarco sia giunto a elaborare la propria versione della vicenda, in cui si trovarono coinvolti Pythes e la "virtuosa" moglie.

Come si è già più volte ribadito, il debito dal dettato erodoteo pare scontato, per quanto assai parziale. Il biografo, che semplifica il racconto del predecessore, sintetizzandolo nel solo secondo segmento della novella 27, introduce ex novo (per quanto ci consta) i segmenti 1 e 3, dei quali non compare traccia nello storico di Alicarnasso, ma neppure in Seneca e in Plinio, suoi diretti predecessori, che si limitano in sostanza a riferire i fatti come riportati da Erodoto. Ciò potrebbe indurre a pensare che il biografo abbia recepito più tradizioni preesistenti, relative alla vicenda di Pythes, ri-assemblandole in modo personale, come richiedeva il contesto narrativo, destinato a contenere la storia. Ma, in via di principio, non sarebbe neppure da escludere che Plutarco possa aver desunto in modo meccanico e indipendente da Erodoto l'intero racconto, rintracciandolo in forma già elaborata in una fonte a noi ignota. E, a rigore, neppure che possa, di persona, aver creato il contenuto dei segmenti 1 e 3, adeguandone i dettagli narrativi a quelli ricorrenti nel segmento centrale, di ispirazione erodotea.

Tuttavia, a ben vedere, quest'ultima ipotesi pare irrealistica. Come è stato dimostrato dalla critica, «the big invention is not in his style ${ }^{69}$; p piuttosto il biografo era solito limitarsi ad arricchire le proprie fonti, componendo di proprio pugno brevi inserti narrativi, verosimili e coerenti con l'assunto complessivo. Ma pure la precedente

67 Sui rapporti di parentela fra Clea e Memmia Leontide, vd. B. Puech, Prosopographie des amis de Plutarque, in ANRW 2, 33, 6, 1992, p. 4831-4893, 4857-4859, con ulteriore bibliografia.

68 Proprio la grande cultura di Clea e di Leontide giustifica il taglio erudito dello scritto, caratterizzato da disquisizioni antiquarie di nicchia, come tiene a rimarcare lo stesso Plutarchus, Moralia, 243D.

69 In tal senso si esprime (p. 153), relativamente alle Vite, C. Pelling, «Truth and Fiction in Plutarch's Lives», in ID. Plutarch and History, p. 143-170, con documentata ed equilibrata discussione del problema. Sostanzialmente non dissimile il punto di vista espresso sul Mulierum virtutes da Boulogne, Plutarque. Conduites, p. 38: " [Plutarque] traite l'histoire non pas en compilateur d'anthologies, mais à la fois en amateur passionné d'histoire qui connaît les œuvres de nombreux historiographes, très connus ou moins célèbres, en historien lui-même critique, en conteur, mais aussi en autheur authentique, qui personnalise ce qu'il emprunte». Sulla rielaborazione plutarchea delle proprie fonti nel Mulierum virtutes vd. anche G. Marasco, «Sul Mulierum virtutes di Plutarco», in D’Ippolito, Gallo (edd.), Strutture formali, p. 335-345, part. p. 333. 
ipotesi risulta destituita di solido fondamento; Plutarco, come è ben noto, non solo fu fine conoscitore di Erodoto, ma fu anche fornito di una cultura storica così ampia da non trovarsi di certo costretto ad estrarre i propri testi meccanicamente da autori, o, peggio, da compilazioni preesistenti. Pertanto pare assai più attendibile prefigurarsi che egli abbia operato una ri-elaborazione personale di racconti popolari da lungo tempo ben noti, verosimilmente presenti in fonti letterarie di area micro-asiatica, come è stato suggerito dalla critica ${ }^{70}$, forse fonti di nicchia, per noi perdute ${ }^{71}$. Del resto è ben noto che l'autore avesse compiuto numerose letture preparatorie alla stesura delle Vite ${ }^{72} \mathrm{e}$ che di tali letture il Mulierum virtutes possa essere considerato prodotto di risulta.

Peraltro, a comprovare indirettamente l'esistenza di più versioni sulla vicenda di Pythios/Pythes, potrebbe concorrere, per analogia, la pluralità di tradizioni, in questo caso a noi pervenute, sulla non dissimile e ben nota vicenda di Gige e di Candaule. Infatti sull'episodio, oltre a Erodoto, ebbero a soffermarsi Xanto di Lidia, Platone e verosimilmente un autore tragico non identificabile ${ }^{73}$. Il contenuto complessivo e la datazione di quest' ultimo testo, giunto in forma assai frammentaria, è di fatto impossibile, così che risulta arduo stabilire se lo storico di Alicarnasso possa aver desunto dalla tragedia, o, al contrario, se possa esserne la fonte. Resta invece assodato che le versioni riferite da Xanto e da Platone, improntate alle leggende popolari, differissero nettamente da quella erodotea, più complessa, intrigante e destinata a veicolare le riflessioni morali dell'autore.

Considerazioni analoghe potrebbero essere avanzate, pur con la dovuta cautela, anche a proposito della storia di Pythios/Pythes ${ }^{74}$. Sarebbe lecito, infatti, inferire che

70 Così, fra gli altri, STADTER, Plutarch's Historical Methods, p. 123. Si ricordi che nel logos 18, dedicato a Lampsaké e di ambientazione micro-asiatica, è evocata la testimonianza di Carone di Lampsaco.

71 Questa ipotesi trova conforto in quanto il medesimo autore, rivolgendosi alla destinataria

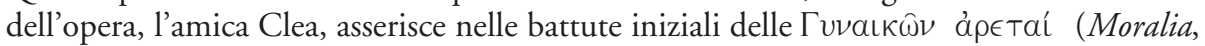
243D) : «Non tratterò i fatti più noti e tutti quelli che credo tu conosca attraverso solide letture, a meno che certi dettagli degni di essere letti non siano sfuggiti a quanti hanno in precedenza riferito queste storie di pubblico dominio».

72 Sul metodo di lavoro plutarcheo la bibliografia è vastissima; fra i contributi più recenti si segnala P. PAYEN, «Si l'on supprime les hommes d'action, on n'aura plus d'hommes de lettres. Thucydide et Polybe dans le corpus des œuvres de Plutarque», in Malosse, NoëL et Schoule (edd.), Clio, p. 13-25.

73 Herodotus I, 7-13; Xanthus Lydus, FGrHist 90 F 47; Plato, Respublica, 359c-360b. Per un sintetico esame delle fonti e per una prima discussione del frammento papiraceo (TrGF II F 664) vd. Saïd, "Herodotus and Tragedy», in Baker, De Jong, van Wees (edd.), Brill's Companion, p. 117-147; Griffin, "Herodotus and Tragedy», in Dewald, Marincola (edd.), The Cambridge Companion, p. 46-59, con discussione della precedente bibliografia.

74 Per certi versi anche la vicenda del Lido potrebbe presentare aspetti tragici, ri-proponendo il tema della presunzione umana e della caduta, della terribile vendetta e persino dell'autosacrificio finale. 
la versione erodotea dei fatti, tutta incentrata sui temi della guerra e dell'oltraggiosa violenza messa in atto dal gran re, costituisse il primitivo nucleo di una storia, in seguito arricchitasi di nuovi dettagli di sapore più romanzesco, orientaleggiante; oppure, al contrario, che lo storico si fosse limitato a estrapolare un segmento narrativo, a lui congeniale, da un preesistente testo della tradizione folclorica popolare ${ }^{75}$, in origine ben più articolato, forse non troppo dissimile dal racconto in seguito proposto da Plutarco.

Purtroppo non disponiamo di elementi utili a suffragare l'una o l'altra ipotesi. Certo sarebbe tentante immaginare (ma soltanto sul filo della fantasia) che, più di cinquecento anni dopo Erodoto, il biografo abbia volutamente concluso il suo trattato sulle Virtù femminili, ri-proponendo e ri-maneggiando, in rapporto antagonistico con lo storico, la versione completa della vicenda di Pythios/Pythes, espressione di un antico folk-tale di impronta micro-asiatica. In tal modo avrebbe ridimensionato, ancora una volta, l'attendibilità documentaria dell'illustre predecessore, che, rinunciando alla veste di teller of tales ${ }^{76}$, si sarebbe limitato a recepire e testimoniare solo i dettagli bellici della medesima storia, arricchendoli, però, di amare valutazioni moralistiche, così da elaborare un testo senz'altro povero di risvolti evenemenziali romanzati, ma ben più ricco di valenze meta-storiche, del tutto estranee alla piana esposizione plutarchea.

Gabriella VANOTTI

Dipartimento di Studi umanistici

Università degli Studi del Piemonte orientale

Via Manzoni 8

I-13100 - Vercelli

(gabriella.vanotti@lett.unipmn.it)

75 Da ultima in tal senso si esprime THOMAS, «Herodotus», p. 238: «It therefore seems probable that there are further stages of tradition and storytelling to be considered in the creation of this tale of Pythius".

76 La stesura delle Storie costituirebbe da parte di Erodoto una rottura definitiva con il suo passato di oral teller of tales secondo W. RöSLER, "The Histories and Writing», in BAKER, De Jong, van Wees (edd.), Brill's Companion, p. 79-94, part. p. 85. 